\title{
Targeting interleukin 18 with interleukin 18 binding protein
}

\author{
Charles A Dinarello
}

\begin{abstract}
A novel, constitutively expressed and secreted interleukin 18 (IL18) binding protein (IL18BP) neutralises IL18. IL18BP shares many characteristics with soluble cytokine receptors of the IL1 family in that the protein exhibits specificity for IL18, belongs to the immunoglobulin-like class of receptors and has limited amino acid sequences with those of the IL1 receptor type II. However, unlike soluble cytokine receptors, IL18BP does not have a transmembrane domain and hence is not anchored to the cell membrane. IL18BP is a secreted protein and not cleaved from the cell surface. IL18BP is naturally occurring and was isolated from the urine of healthy subjects. Because IL18 is an important inducer of interferon $\gamma$ (IFN $\gamma$ ), IL18BP suppresses the production of IFN $\gamma$ resulting in reduced T-helper type 1 immune responses. There are four human and two mouse isoforms-resulting from mRNA splicing and found in various CDNA libraries. Each of these IL18BP isoforms have been expressed, purified and assessed for binding and neutralisation of IL18 biological activities. Two human IL18BP isoforms exhibited the greatest affinity for IL18 with a rapid on-rate, a slow off-rate and a dissociation constant ( $\mathrm{kDa}$ ) of 399 pM. The two other isoforms with an incomplete immunoglobulin domain were unable to neutralise IL18. The two human isoforms that possess a complete immunoglobulin domain, neutralise $>\mathbf{9 5 \%}$ IL18 at a molar excess of two. Molecular modelling identified a large mixed electrostatic and hydrophobic binding site in the immunoglobulin domain of IL18BP, which could account for its high affinity binding to the ligand. These high affinity forms may be ideally suited for blocking IL18 in human disease. It is likely that preferential secretion of high affinity functional and nonfunctional isoforms of IL18BP affect the immune response and the outcome of disease.

(Ann Rheum Dis 2000;59(suppl I):i17-i20)
\end{abstract}

University of Colorado Health Sciences Center, Division Infectious Diseases, B168, 4200 East Ninth Avenue, Denver, CO 80262, USA

Correspondence to: Dr Dinarello

(charles.dinarello@uchsc.edu)
Immune responses to antigens are divided into T lymphocyte helper type 1 (Th1) and type 2 (Th2). ${ }^{1}$ Th1 responses include the secretion of cytokines interleukin (IL) 2, IL12 and interferon $\gamma(\mathrm{IFN} \gamma)$ and the generation of specific cytotoxic $\mathrm{T}$ lymphocytes recognising specific antigen. The Th2 response is characterised by the cytokine IL 4 and the production of specific antibodies to the antigen. The Th1 response is a vital arm of host defence against many organisms; however, the Th1 response is also associ- ated with several autoimmune diseases as well as organ transplant rejection. Therefore, natural regulation of the Th1 cytokines probably affects the intensity of the Th1 response.

IL18, originally termed IFN $\gamma$ inducing factor, ${ }^{2}$ is a Th1 cytokine. Mice deficient in IL1 8 have a blunted natural killer cell response and markedly reduced IFN $\gamma$ production. ${ }^{3}$ IL 12 upregulates the IL18 receptor $\alpha$ chain, ${ }^{4}$ a member of the IL1 receptor family. ${ }^{56}$ In fact, this regulation of the IL18 receptor may explain the well established synergism of IL18 plus IL12 in the production of IFN $\gamma$. Although an inducible homodimer of the IL12 p40 chain can act as a receptor antagonist and inhibit IL12, there is no evidence that this dimer is expressed in humans, and therefore there is no constitutive inhibitor of IL12 described to date. On the other hand, a novel IL1 8 binding protein (IL18BP) has been described ${ }^{7}$ and being constitutively expressed and secreted, binds IL18, acts as a natural inhibitor of IL18 induced IFN $\gamma$ and suppresses the Th1 response. The IL18BP seems to be the only constitutively secreted protein that impacts upon the Th1 response after infection or immune stimulation. Because IL18BP is a natural product, it is an attractive treatment for treating diseases that mediated, in part, by IL12, IFN $\gamma$ or IL18 itself.

With a single immunoglobulin (Ig) domain, IL18BP resembles the extracellular segment of cytokine receptors with Ig-like structures; however, IL18BP is a novel protein distinct from the IL1 and IL18 receptor families. ${ }^{7}$ Located in chromosome 11q13 at the inverted position of the nuclear mitotic apparatus protein-1, the human IL18BP gene encodes for at least four distinct isoforms (IL18BPa, b, c and d) derived from $\mathrm{mRNA}$ splice variants isolated from several cDNA libraries. Two isoforms of murine IL18BP were similarly cloned. Little is known about the ability of these six isoforms of IL18BP to bind and neutralise IL18. They differ primarily in their carboxyl termini whereas the $\mathrm{N}$-terminal one third to two thirds of the amino acids are identical. If these isoforms vary significantly in their ability to neutralise the biological activity of IL 18 , one may conclude that the Th1 response will be affected in people expressing one isoform preferentially compared with another isoform.

\section{Neutralisation of IL18 activity by different IL18BP isoforms}

Six purified isoforms of IL18BP were tested for inhibition of human IL18 at different molar ratios of IL18BP to human IL18. Complete inhibition of IL18 activity on natural killer cells was observed at a twofold molar excess of 
IL18BPa over IL18. At equimolar ratios, inhibition by IL18BPa was approximately $50 \%$. Equimolar and molar excess of two of human IL18BPc exhibited similar potency as IL $18 \mathrm{BPa}$. In contrast, there was no inhibition of IL18 activity by IL $18 \mathrm{BPb}$ or $\mathrm{d}$ at a molar excess of two. Higher ratios of sixfold molar excess of these isoforms also lacked inhibitory activity. $^{8}$

\section{Modelling of IL18BP with IL18}

Sequence alignment of IL18BPa was assessed against two possible modelling templates: domain III of IL1R and domain 1 of CD $4,{ }^{9}$ which shows $27.6 \%$ sequence identity with the same fragment in IL18BP. The templates and IL18BP carry several established identifiers of the Ig fold, including the conserved cysteines and tryptophan. The final alignment of IL18BP against IL1R type I was made. ${ }^{8}$ IL18 was aligned to IL1 $\beta$ as described previously, ${ }^{10}$ but with modifications, in particular at the $\mathrm{N}$ and $\mathrm{C}$-terminals. The final sequence alignment of IL1 8 against human-IL $\beta$ was made.

\section{The interface of IL18 with IL18BP}

IL18BP interacts with IL18 through $\beta$-strands. The model of IL18BP is reliable for most of the sequence including the fragments that interact with IL18. IL18 interacts with IL18BP via residues $85,87,89,130,132,143$ and 149 , which are found in the sequence fragment that could be reliably aligned with IL $1 \beta$, but also with the $\mathrm{N}$ - and $\mathrm{C}$-terminal $\beta$-strands (residues 42 and 189). The model suggests that the interaction of IL18BP and IL18 is stabilised, on the one hand, by strong electrostatic interactions (buried ion pairs between E42 in IL1 8 and K130 in IL18BP and between K89 in IL18 and E114 in IL18BP) and also by the burial of large hydrophobic patches. The latter consist of residues I85, M87, M96, P143, M149 and L189 in IL18 and F93, I95, Y97, V151 and P153 in IL18BP. Notably, one of the ion pairs is less reliable because it involves E42 in IL18, which is found in the N-terminal $\beta$-strand, where the alignment is least accurate.

\section{Implications for disease and treatment} Diseases associated with cytokine polymorphisms are mostly defects in the receptors and not the ligands. The finding of the different isoforms of IL18BP in the various libraries may be tissue specific or related to events during cell stimulation. The IL18BPa cDNA was the most abundant clone in each of the human libraries. IL18BPc was present in Jurkat and spleen libraries. IL $18 \mathrm{BPb}$ was found in monocytes and Jurkat libraries and IL18BPd in Jurkat cells only. The findings of the different isoforms in the various libraries may have significance for the Th1 immune response. The clinical significance of this study involves natural regulation of the Th1 response. At present, it seems that IL $18 \mathrm{BPa}$ is the major form constitutively expressed in human spleen. ${ }^{7}$ It is presently unknown whether the other human isoforms are expressed and secreted in vivo. The 40 $\mathrm{N}$-terminal amino acids of the naturally occurring urinary $\mathrm{IL}_{18 \mathrm{BP}^{7}}$ are common to each of the four isoforms. As IL $18 \mathrm{BPb}$ and $\mathrm{d}$ do not bind to immobilised IL18, the existence of these isoforms in human urine is possible.

These studies also show that the ability of different isoforms of IL18BP to bind and inhibit the biological activity of IL18 primarily resides in the Ig domain with a possible contribution of the carboxyl end of the molecule. The ability of human IL18BPa and IL18BPc to neutralise human IL18 at equimolar concentrations supports the concept that the Ig domain possesses the essential binding requirements for IL18. Although the first 97 $\mathrm{N}$-terminal mature amino acids $(58 \%)$ of human IL18BPd are identical to human IL18BPa, IL18BPd lacks a complete Ig domain and is inactive in inhibiting IL18 as well as binding to immobilised IL18. The most relevant crystallographic analysis to this study is of the binding of IL $1 \beta$ to IL1R type I and the importance of the third Ig domain. ${ }^{11}$ In fact, antibodies directed to the third Ig domain of the IL1R type I block binding of IL1 $\beta$ whereas antibodies to the $\mathrm{N}$-terminal domains have no effect. ${ }^{12}$

Both the human IL18BPa and $\mathrm{c}$ isoform inhibited $50 \%$ human IL18BP at equimolar ratios. This is an unusually low molar ratio to observe inhibition of biological activity in a 24 hour bioassay. With soluble receptors such as the IL1 receptor type $\mathrm{I}$ and $\mathrm{II}^{13}$ and the TNF receptor p55 and $\mathrm{p} 75,{ }^{14}$ the molar excess required to inhibit $50 \%$ of biological activity of the respective ligands is at least fivefold. One likely explanation for this potency of IL $18 \mathrm{BPa}$ and $c$ is the high affinity binding and slow offrate of both forms. Molecular modelling identified a large interaction site with IL18, which buries $1170 \AA^{2}$ for both molecules. The site includes two strong electrostatic ion pair interactions (E42 in IL18 and K130 in IL18BP and K89 in IL18 and E114 IL18BP), which are buried near the centre of the interface and are unlikely to be surrounded by water. The binding is further strengthened by the burial of matching hydrophobic patches found on the surfaces of the two molecules.

The first three potential glycosylation sites derived from the primary amino acid sequences of the IL18BP isoforms ${ }^{7}$ were found to be exposed in model structure. Glycosylation of IL18BP may play a part in its function as an inhibitor of IL18 but whether this role is one of solubility or of binding remains unclear. Human IL18BPa and IL18BPc have identical glycosylation sites. In each of the present experiments, ligand passing by IL18BPs as evidence by increased bioactivity was not observed as has been observed with cytokine soluble receptors. ${ }^{14} 15$

$M$ contagiosum viral proteins MC53 and MC54 share a significant homology to mammalian IL18BP. ${ }^{7}$ Recently Moss and coworkers reported that $M$ contagiosum proteins MC53 and MC54 possess the ability to bind and neutralise human IL18 in a fashion similar to that of IL18BP. ${ }^{16}$ As proposed above, the data from the present studies on the mammalian IL18BP isoforms support the hypothesis that the binding and neutralising properties of IL18BP 
reside in the Ig domain. Nevertheless, there is homology in the stretch from 174-191; between $174-189$ of the human IL18BPa there is $83 \%$ homology with viral MC53 and between IL18BPa 176-192, 68\% with viral MC54.

There are many examples of exon splicing events for cytokines and cytokine receptors, but there are few clinical examples of diseases due to these splicing events. Because IL $18 \mathrm{BPb}$ and $\mathrm{d}$ were unable to neutralise IL18, their presence in vivo would probably not have a clinical impact on the Th1 response. However, if some people preferentially undergo the IL $18 \mathrm{BPb}$ and $\mathrm{d}$ splicing events over those of IL $18 \mathrm{BPa}$ and $\mathrm{c}$, then you would expect a reduced ability to inhibit the activity of IL18 during a Th1 response compared with people who secrete the IL $18 \mathrm{BPa}$ and $\mathrm{c}$ isoforms. In the case of IL18BP, two events can result in isoform formation. For example, during routine intron excision, exon skipping takes place resulting in a frame shift and a new carboxyl terminus. The second example also results in a new carboxyl terminus after an in-exon splicing event. In both cases, an early stop codon may contribute to the size of the isoform. In IL $18 \mathrm{BPb}$ and $\mathrm{d}$, in-exon splicing takes place in exon 4 resulting in a new C-terminal amino acid sequence because of a frame shift. As cDNAs for the IL18BPa as well as IL $18 \mathrm{BPb}$ were found in a human blood monocyte library, it is possible that some people may preferentially produce one isoform over the other. Control of in-exon splicing is poorly understood, but in the case of IL18BP, the mechanism for in-exon splicing may have significant clinical impact.

\section{Diseases that may benefit from IL18BP treatment}

As shown in the box, several diseases may benefit from IL18BP treatment. These studies are based on data using neutralising antibodies to IL18, ICE deficient mice ${ }^{17}$ and IL18 or IL18R deficient mice. ${ }^{18}$ In each case, you must consider the suppression of IFN $\gamma$ in potential mechanism of action of blocking the activity of IL18. For example, the use of IL18BP to treat patients with lupus erythematosus is based on the pathogenic role of IFN $\gamma$ in this disease. However, in other models using salmonella endotoxin ${ }^{19}$ or toxin induced hepatitis, ${ }^{20}$ there is no role for IFN $\gamma$ and in these models, blocking IL18 seems to be the sole mechanism.

In the case of treating Crohn's disease, you must be cautious about the role of prostaglan-

\section{Possible diseases for therapeutic use of IL18BP}

Rheumatoid arthritis

Inflammatory bowel disease (Crohn's disease)

Toxic liver damage

Lupus erythematosus

Still's disease

Psoriasis

Graft versus host

Transplant rejection

Multiple sclerosis

Septic shock din (PG) $\mathrm{E}_{2}$ and IFN $\gamma$ in this disease. IL18 shares with IL1 the same family of receptors and several identical signal transduction pathways. Because of these similarities, IL18 may induce $\mathrm{PGE}_{2}$ synthesis. However, in human peripheral blood mononuclear cells (PBMC), a prominent, proinflammatory property of IL 1 is $\mathrm{PGE}_{2}$ production. Although IL18 was highly active in PBMC by inducing the synthesis of the chemokine IL8, no induction of $\mathrm{PGE}_{2}$ synthesis nor cyclooxygenase type 2 gene expression was observed in PBMC stimulated with IL18. In the same cultures, IL1 $\beta$ induced a 12 -fold increase in $\mathrm{PGE}_{2}$. In fact, IL1 $\beta$ induced IL8 synthesis was augmented threefold by IL18, but IL1 8 suppressed IL1 $\beta$ induced $\mathrm{PGE}_{2}$ production by $40 \%{ }^{21}$ The suppressive effect of IL18 on PGE2 production was mediated by IFN $\gamma$ as antihuman IFN $\gamma$ antibody prevented IL1 8 induced reduction in $\mathrm{PGE}_{2}$.

In rats, administration of COX inhibitors increase immune mediated colitis. ${ }^{22}$ As a potent immunomodulator, $\mathrm{PGE}_{2}$ promotes Th2 responses in dendritic and Th0 cells ${ }^{23}$ and inhibits the production of Th1 lymphokines by T cells. $\mathrm{PGE}_{2}$ also downregulates expression of the IL12 receptor, decreases responsiveness of human PBMC to IL $12^{25}$ and reduces the production of IL12 and IFN $\gamma$ in PBMC. ${ }^{26}$ Therefore, the fact that IL18BP increases both spontaneous and IL1 $\beta$ induced $\mathrm{PGE}_{2}$ production supports the concept that IL18 and IL12 from freshly isolated PBMC affects $\mathrm{PGE}_{2}$ production in these cells via IFN $\gamma$. As IL18BP is constitutively expressed in humans ${ }^{27}$ its role in $\mathrm{PGE}_{2}$ synthesis may affect the progression and severity of autoimmune diseases, particularly in inflammatory bowel disease.

Funding: these studies are supported by NIH Grant A-15614, AI-2532359, Colorado Cancer Center CA 46934 (to CAD).

1 Seder RA, Paul WE. Acquisition of lymphokine-producing phenotype by CD4+ $\mathrm{T}$ cells. Annu Rev Immunol

2 Nakamura K, Okamura H, Wada M, Nagata K, Tamura T. Endotoxin-induced serum factor that stimulates gamma inteferon production. Infect Immun 1989;57:590-5.

3 Takeda K, Tsutsui H, Yoshimoto T, Adachi O, Yoshida N, Kishimoto K, et al. Defective NK cell activity and Th1 response in IL-18-deficient mice. Immunity 1998;8:38390.

4 Yoshimoto T, Takeda K, Tanaka T, Ohkusu K, Kashiwamura S, Okamura H, et al. IL-12 upregulates IL-18 receptor expression on $\mathrm{T}$ cells, Th1 cells and B cells: synergism with IL-18 for IFN $\gamma$ production. J Immunol 1998;161: 3400-7

5 Parnet P, Garka KE, Bonnert TP, Dower SK, Sims JE. IL-1Rrp is a novel receptor-like molecule similar to the type I interleukin-1 receptor and its homologues T1/ST2 and IL-1R AcP. J Biol Chem 1996;271:3967-70.

6 Torigoe K, Ushio S, Okura T, Kobayashi S, Taniai M, Kunikate T, et al. Purification and characterization of the human interleukin-18 receptor. J Biol Chem 1997;272: human inter.

7 Novick D, Kim S-H, Fantuzzi G, Reznikov L, Dinarello CA, Rubinstein $M$. Interleukin-18 binding protein: a novel modulator of the Th1 cytokine response. Immunity 1999;10:127-36.

8 Kim S-H, Eisenstein M, Reznikov L, Fantuzzi G, Novick D, Rubinstein $\mathrm{M}$, et al. Structural requirements of six naturally occurring isoforms of the interleukin-18 binding protein to inhibit interleukin-18. Proc Natl Acad Sci USA 2000;97:1190-5.

9 Miki T, Matsui T, Heidaran M, Aaronson SA. An efficient directional cloning system to construct cDNA libraries containing full -length inserts at hight frequency. Gene 1989;83:137-46.

10 Bazan JF, Timans JC, Kaselein RA. A newly defined interleukin-1? Nature 1996;379:591.

11 Vigers GPA, Anderson LJ, Caffes P, Brandhuber BJ. Crystal structure of the type I interleukin-1 receptor complexed structure of the type I interleukin-1 receptor

12 Clark BD, Ikejima T, Mancilla J, Sirko S, Orencole SF, Isji $\mathrm{N}$, et al. An antibody to a 17 amino acid synthetic peptide 
to the IL-1RI preferentially blocks IL-1 $\beta$ binding. J Interferon Cytokine Res 1996;16:1079-88.

13 Arend WP, Malyak M, Smith MF, Whisenand TD, Slack JL, Sims JE, et al. Binding of IL- $1 \alpha$, IL-1 $1 \beta$, and IL- 1 receptor tors and levels of soluble IL-1 receptors in synovial fluids. J Immunol 1994;153: 4766-74

14 Terlizzese M, Simoni P, Antonetti F. In vitro comparison of inhibiting ability of soluble TNF receptor p75 (TBP II) vs. soluble TNF receptor p55 (TBP I) against TNF-alpha and TNF-beta. J Interferon Cytokine Res 1996;16:1047-53.

15 Aderka D, Engelmann H, Maor Y, Brakebusch C, Wallach D. Stabilization of the bioactivity of tumor necrosis factor by its soluble receptors. J Exp Med 1992;175:323-9.

16 Xiang Y, Moss B. IL-18 binding and inhibition of interferon gamma induction by human poxvirus-encoded proteins. Proc Natl Acad Sci USA 1999;96:11537-42.

17 Vidal-Vanaclocha F, Fantuzzi G, Mendoza L, Fuentes AM, Anasagasti MJ, Martin J, et al. IL-18 regulates IL-1betadependent hepatic melanoma metastasis via vascular cell dependent hepatic melanoma metastasis via vascular cell 734-9.

18 Hoshino K, Tsutsui $\mathrm{H}$, Kawai T, Takeda K, Nakanishi $\mathrm{K}$, Takeda Y, et al. Cutting edge: generation of IL-18 receptoras an essential IL-18 binding receptor. J Immunol 1999;162:5041-4

19 Netea MG, Fantuzzi G, Kullberg BJ, Stuyt RJ, Pulido EJ, McIntyre RC, Jr, et al. Neutralization of IL-18 reduces neutrophil tissue accumulation and protects mice against lethal Escherichia coli and Salmonella typhimurium endotoxemia. J Immunol 2000;164:2644-9.
20 Faggioni R, Jones-Carson J, Reed D, Dinarello C, Feingold $\mathrm{K}$, Grunfield $\mathrm{C}$, et al. Leptin-deficient (ob/ob) mice are protected from t cell-mediated hepatotoxicity: role of USA 2000;97:2367-72.

21 Reznikov LL, Kim SH, Westcott JY, Frishman J, Fantuzzi $\mathrm{G}$, Novick D, et al. IL-18 binding protein increases spontaneous and IL-1-induced prostaglandin production via inhibition of IFN-gamma. Proc Natl Acad Sci USA 2000;97: 2174-9.

22 Reuter BK, Asfaha S, Buret A, Sharkey KA, Wallace JL. Exacerbation of inflammation-associated colonic injury in rat through inhibition of cyclooxygenase-2. J Clin Invest 1996;98:2076-85.

23 Gold KN, Weyand CM, Goronzy JJ. Modulation of helper T cell function by prostaglandins. Arthritis Rheum 1994;37: 925-33.

24 Kalinski P, Hilkens CM, Snijders A, Snijdewint FG Kapsenberg ML. Dendritic cells, obtained from peripheral blood precursors in the presence of PGE2, promote Th2 responses. Adv Exp Med Biol 1997;417:363-7.

25 Wu CY, Wang K, McDyer JF, Seder RA. Prostaglandin E2 and dexamethasone inhibit IL-12 receptor expression and and dexamethasone inhibit IL-12 receptor expression

26 Demeure CE, Yang LP, Desjardins C, Raynauld P, Delespesse G. Prostaglandin E2 primes naive T cells for the production of anti- inflammatory cytokines. Eur J Immunol 1997;27:3526-31.

27 Puren AJ, Fantuzzi G, Dinarello CA. Gene expression, synthesis and secretion of IL-1 $\beta$ and IL-18 are differentially regulated in human blood mononuclear cells and mouse pleen cells. Proc Natl Acad Sci USA 1999;96:2256-61. 